\title{
Digital Assisted Image Correlation for Metal Sheet Strain Measurement
}

\author{
García-Alcalá Carlos-Eduardo $^{1(\otimes)}$ (D), Padilla-Medina José-Alfredo ${ }^{1(\otimes)}(\mathbb{D})$, \\ and Barranco-Gutiérrez Alejandro-Israel ${ }^{1,2(\bowtie)}$ (D) \\ 1 TecNM en Celaya, 38010 Guanajuato, Mexico \\ cgalcala92@gmail.com, \\ \{alfredo.padilla, israel.barranco\}@itcelaya.edu.mx \\ 2 Cátedras CONACyT, 03940 Ciudad de México, Mexico
}

\begin{abstract}
Current methods of correlation and point matching between stereoscopic images produce large errors or are completely inefficient when the surface has a repetitive, non-isotropic, low contrast pattern. In this article a new method of Digital Assisted Image Correlation (DAIC) is presented to match specific points in order to estimate the deformation of the surface in the metal sheets used in the automotive industry. To achieve this, it is necessary to stamp the surface to be measured with a regular pattern of points, then a digital image processing is done to obtain the labels of the circles of the pattern. After this, a semi-automatic search is made in the labels of both images to correlate all of them and perform the triangulation. DIC is used to corroborate the correspondence between points and verify the accuracy and efficiency of the developed method. This allows the $3 \mathrm{D}$ reconstruction of the sheet with a minimum of information and provides more efficiency and a great benefit in computational cost. Deformation is calculated by two methods, which show similarity between the values obtained with a digital microscope. It is assumed that quality of marks stamping, lighting, and the initial conditions, also contribute for trustworthy effects.
\end{abstract}

Keywords: Image matching $\cdot$ Steel industry $\cdot$ Stereo vision $\cdot$ Digital Image Correlation

\section{Introduction}

The deformation measurement in metal sheets is a very important activity to know the material properties used to manufacture them, to assure the quality and applications design where is used, in this case, the automotive industry [1]. Commonly, multicamera vision has been used to measure it, especially stereoscopic vision [2]. The surface deformation measurement of the metal sheets depends on the $3 \mathrm{D}$ points reconstruction efficiency from a stereoscopic digital camera system and the latter is subject to points matching. One of the most popular methods in mechanics to strain estimation is called Digital Image Correlation (DIC). This is a full-field non-contact optical-numerical technique to measure shape, motion, and deformation, on almost any kind of material, even in extreme experimental settings [3], as long as the region of interest (ROI) on the sample's 
surface is provided with a natural or synthetic speckle pattern [4]. This allows finding the best match between corresponding points in the two images by comparing the local grey scale distribution of square pixel subsets on the basis of the normalized cross correlation coefficient. The uniqueness of each signature is only guaranteed if the surface has a non-repetitive, isotropic, high contrast pattern [5, 6] (Fig. 1). An efficient matching operation requires the two images to be similar in terms of speckle pattern appearance. Extracting information from such images is particularly challenging because the success of a DIC matching is guaranteed only when a sufficient similarity exists between the images to be correlated [7-9]. This can be achieved by using a pair of 'twin' cameras (with identical settings) and proper illumination, or by capturing both views simultaneously with one single camera and additional external optical devices [10]. Nonetheless, the use of DIC data to validate models in a quantitative way or to identify with precision several constitutive parameters, remains not easy work [11]. One of the reasons is the complicate compromise between the measuring resolution and the large space for measuring to be done. A second reason is the state of frontiers. A third reason is that measured displacements are not directly compared with simulations.
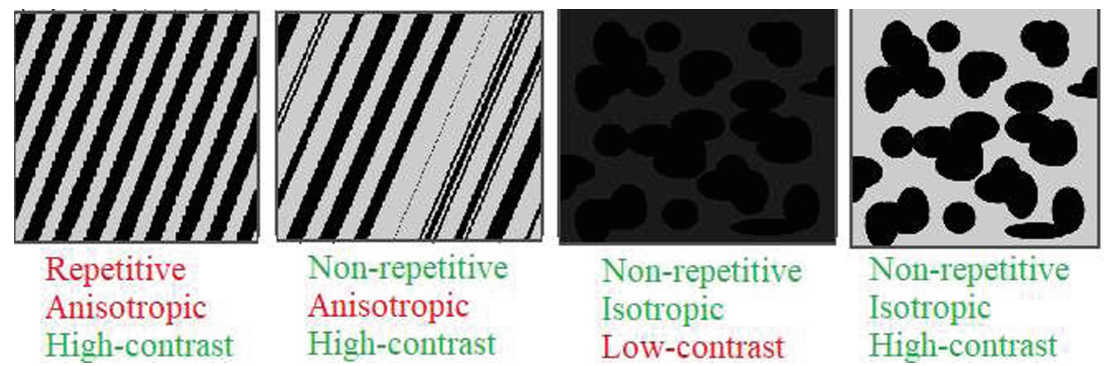

Fig. 1. Image specifications to implement DIC correctly [12].

On the other hand, the grid of circles as references to measure deformation in metal sheets, is widely used [13-16, 29] because the accuracy and precision of the measurements can be analyzed manually with a microscope. Figure 2 shows the deformation phenomenon of the metal sheets and what happens with the stamped marks, as the distance between the centroids changes.

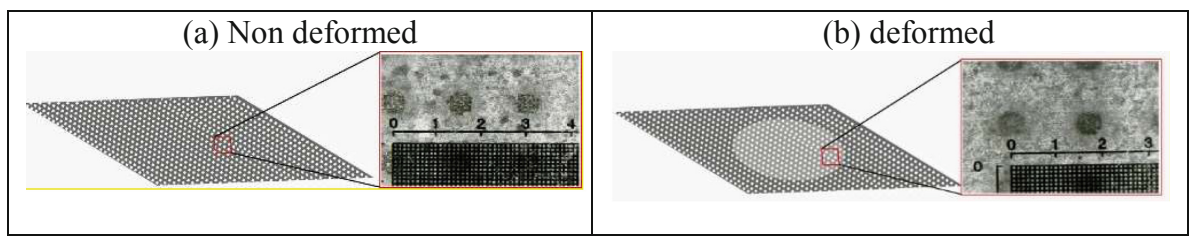

Fig. 2. (a) Circles on metal sheet without deformation; (b) Circles on deformed metal sheet. 
There are several efforts presenting promising results like [8, 11, 17-25]. In ref. [18] they propose a deconvolution algorithm that allows to eliminate, over a certain frequency range, the systematic error for DIC and LSA (Localized Spectrum Analysis). The procedure diminishes the value of the spatial resolution (thus improves it). The drawback is that the noise level impairing the measurements increases, but not in the same proportion as the decrease in spatial resolution. Hence the compromise between measurement resolution and spatial resolution is significantly improved, which makes this deconvolution procedure potentially useful in situations where localized strain gradients occur. In Ref. [19] they proved a new method called Simplified Digital Image Correlation Method (SiDIC) in a rubber balloon to verify if the super pression aerostatic balloon could be measured. The SiDIC correctly identified the non-deformed region, although the deformation is not accurate. In Ref. [11] it is proposed the use of an initial condition which consist in doing click on four points of circles grid to accelerate the marks searching and improve the points matching efficiency. It is observed that the errors of measured displacements using DIC are closely related to the quality of the speckle pattern [21, 24-26]. In other words, the measured displacements of different speckle patterns using the DIC technique may be different even though the deformation state of the specimen, the calculation parameters (e.g., the correlation criteria, sub-pixel registration algorithm, subset size, subset shape function, the interpolation scheme, and calculation path) are the same. Nowadays DIC is often used in a qualitative manner rather than as a metrological tool. This is especially due to the time-consuming task related to the post-processing of the images: in Ref. [27] more than 10000 points were exported from the camera measurements.

In this work, we propose a new two-point initialization technique to improve velocity and accurate processing in 3D reconstruction and the calculation of surface deformation in a metallic sheet. This is done by stamping a grid of circular oxide dots on the metal sheet. After obtaining the corresponding labels of each point, a semi-automatic algorithm of coincident points between both images is implemented. The factor correlation is used to verify point matching. Subsequently, the deformation is calculated by the average distance of four neighbors, finally the measurement is compared with that obtained with a digital microscope.

\section{Materials and Methods}

In order to capture the images a pair of Prosilica GT2750 cameras were used. These are 6.1 megapixels each, with a Gigabit Ethernet port compatible with GigE Vision and a Hirose I/O port. This kind of camera incorporates a high-quality Sony sensor ICX694 EXview HAD CCD that provides extra sensitivity, near IR response, low noise level, antiblooming effect and excellent image quality. At full resolution, this camera processes 19.8 frames per second. With a smaller region of interest, higher frame rates are possible. It is a robust device designed to operate in extreme environments and fluctuating lighting conditions. It offers precise control of the iris lens that allows users to set the size of the aperture to optimize depth of field, exposure and gain without the need for additional control elements. For the assembly, a metal structure of $40 \times 40 \times 40 \mathrm{~cm}$ was designed, the piece to be measured is approximately at $30 \mathrm{~cm}$ from the lens of the cameras. LED 
lighting was chosen due to the contrast with the circles marked on the sheet: blue LED of $640 \mathrm{~nm}$. The acquired images have a resolution of $2752 \times 2200$ pixels. To compare the dimensional measurements, a digital microscope (Jiusion 6-06814-24289-8) with a scale of $100 \mu \mathrm{m}$ was used (Fig. 3).

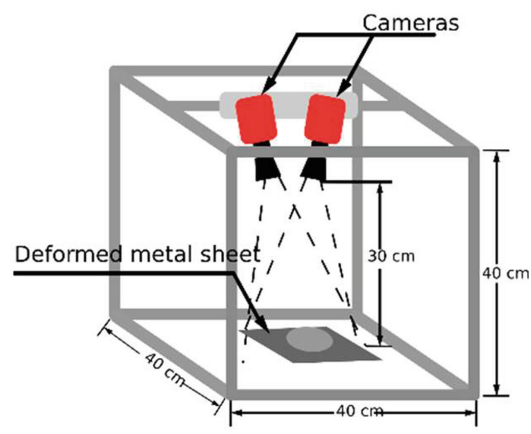

Fig. 3. Configuration of stereo cameras, with lighting and the metal sheet.

The followed methodology has the purpose of measuring the surface deformation in a metal sheet used for car bodies.

1. Calibrations of the cameras.

2. Stamping of known circle grid on the not deformed metallic sheet.

3. Deformation of the metal-sheet by the mechanical stamping process.

4. Illumination of the piece with LED blue light for being measured.

5. Capture of stereo images.

6. Digital image processing to obtain the labels of the points of the metal sheet.

7. Manual selection of the same point in both images to allow the algorithm to matching all the remaining points among them.

8. Triangulation of points to obtain their position in 3D space and reconstruction of the metal sheet.

9. Estimate the deformation by two methods:

- From the average distances of each point with its neighbors in 3D space.

- Through the depth value of each point (measurement on $\mathrm{Z}$ axis).

For the individual and stereo calibration of the cameras, the defined functions provided by the OpenCV library were used within the Python programming language. The process includes the stamping of the grid of points in the metal sheet without deforming, this involves an electrochemical process in which an electrolyte is applied as reagent on the surface of the sheet to engrave a thin pattern so that when the sheet is painted, the marking is imperceptible. After the sheet is subjected to the inlaying process, the circles are distorted in the form of ellipses and the distances between the centroids of these are modified, which are used in the digital images to determine the deformation states in the testing metal sheet. 


\subsection{Two Points Labels-Matching Initializations}

After obtaining the first six steps of the methodology as detailed in the previous work [11], they have the right and left labeled images as shown in Fig. 4. In these images each label corresponds to one mark of the dot pattern. The noise was eliminated by filtering the labels by areas so that only those corresponding to the dot pattern remained.

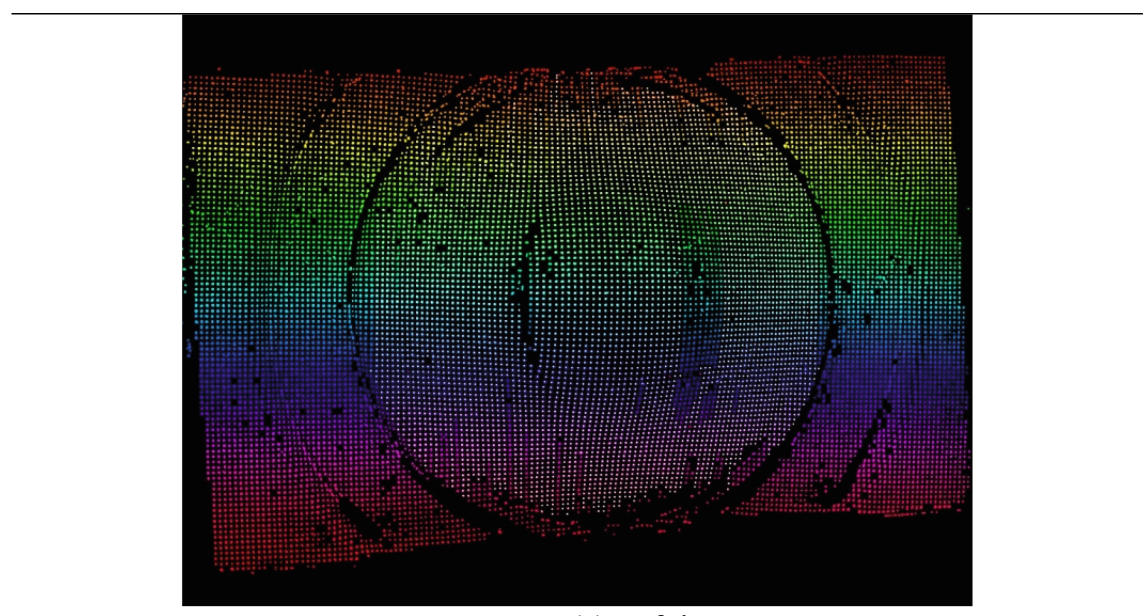

(a) Left image

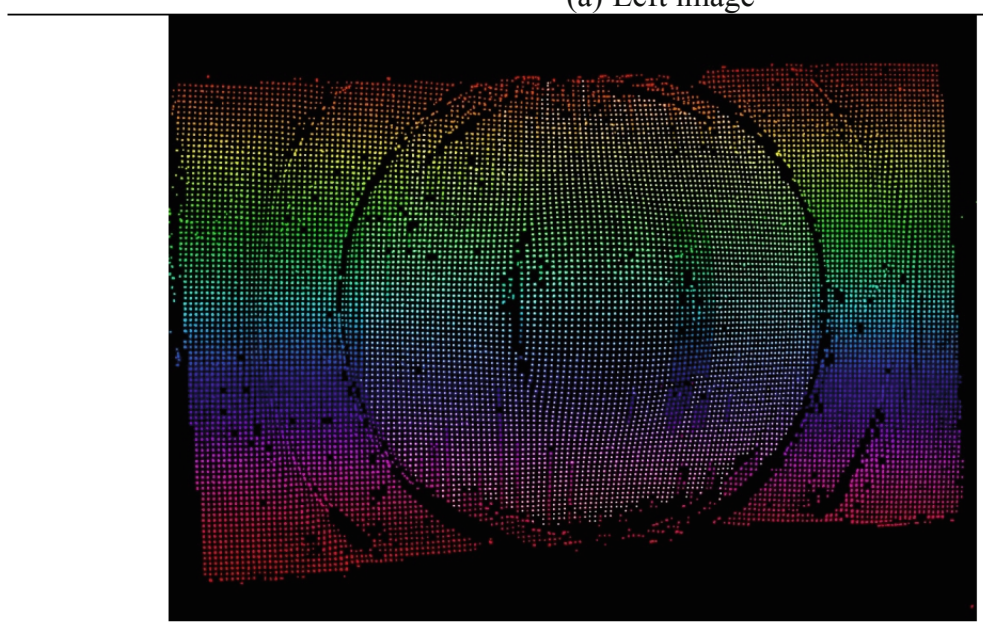

(b) Right image

Fig. 4. Labeled images from stereo cameras after image processing process

The method's aim is to determine the correspondences between 2D centroid coordinates of both images for each circle of the grid pattern on the metal piece giving only one click on each image (left and right). First a click is given on any circle on the image taken by a left camera, then another click is given at that same circle but on the other image taken by the right camera. With this, the centroid of the labels corresponding to 
these marks is determined and the first correspondence between both images is obtained manually (red circle in Fig. 5a). Then a search of neighboring labels in areas near the central circle is made, as shown in Fig. 5a, here are observed in yellow boxes the regions where tags are searched in both images. A new correspondence is obtained if both labels are found in the corresponding boxes of both images. The process is iterative, taking as a central point each new matched label correctly.

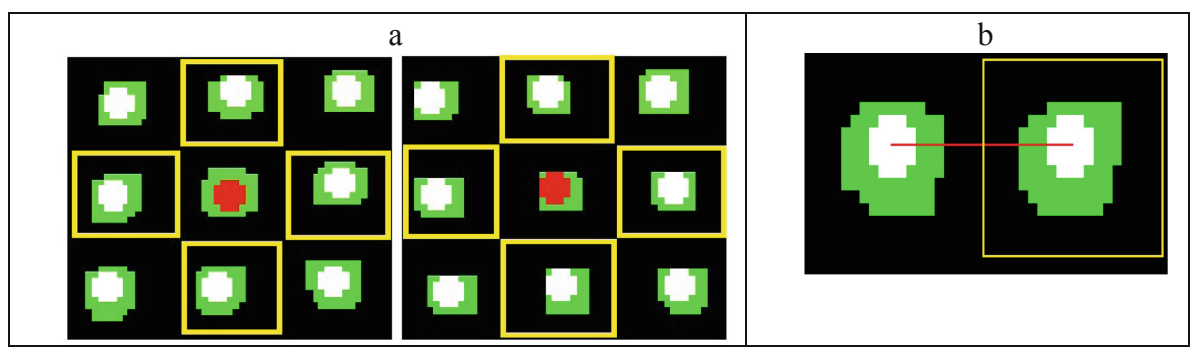

Fig. 5. (a) Semi-automatic matching of labels between both images. (b) Delimiting the area for searches of neighboring labels.

The dimensions of each region of 2D space where each neighboring label is searched and its exact location in space depend on the configuration and size of the dot pattern. One way to do this would be by calculating the distance and the slope between the central point and a neighbor found.

$$
\begin{gathered}
d_{a \leftrightarrow b}=\sqrt{\left(y_{a}-y_{b}\right)^{2}+\left(x_{a}-x_{b}\right)^{2}} \\
m_{a \leftrightarrow b}=\frac{\left(y_{b}-y_{a}\right)}{\left(x_{b}-x_{a}\right)}
\end{gathered}
$$

In Eqs. (1) and (2) the coordinates of the centroids of the labels are used. An imaginary square will be constructed to delimit the search area of each neighboring label (Fig. 5b), each side will have a length equal to the segment $d(a-b)$, one side of the square will cut it perpendicularly at its midpoint. With this the remaining square is built, within which it will be verified pixel by pixel if there is a label.

\subsection{Verify the Point-Pairing Through DIC}

2D-DIC is used to correlate a given set of points in the two stereo views of the reference configuration and match these points along the sequence of images. The Fourier methods can be used to calculate the correlation in a fast way. Equation (3) presents the definition of normalized cross correlation.

$$
N C C=\frac{\sum_{(i, j) \varepsilon s}\left[f_{(u, v)}-\bar{f}\right][g(u, v)-\bar{g}]}{\sqrt{\sum_{(i, j) \varepsilon s}\left[f_{(u, v)}-\bar{f}\right]^{2} \sum_{(i, j) \varepsilon s}[g(u, v)-\bar{g}]^{2}}}
$$

Here $f$ and $g$ are each the grayscale functions of the windows of the current image at a specific location (x, y). The functions $\bar{f}$ and $\bar{g}$ correspond to the gray scale mean of the reference image and the current subset. 


\subsection{Triangulation and 3D Reconstruction}

In Ref. [22] was described the dimensional estimation using stereoscopic vision systems. They quantitatively concluded that the accuracy and precision depend on the distance between the camera and the object to be measured, as well as the resolution of the cameras used. Stereoscopic vision is a technique frequently used to locate points in three dimensions (3D) based on points in two or more 2D images [10, 11, 27-33]. It is necessary to calibrate both cameras using the PinHole model which is described by Eq. (4).

$$
s \tilde{m}=A[R \mid \mathrm{t}] \tilde{M}=\left[\begin{array}{ccc}
f_{x} & 0 & u_{0} \\
0 & f_{y} & v_{0} \\
0 & 0 & 1
\end{array}\right]\left[\begin{array}{ccc|c}
r_{11} & r_{12} & r_{13} \mid & \mathrm{t}_{\mathrm{x}} \\
r_{21} & r_{22} & r_{23} \mid & t_{y} \\
r_{31} & r_{32} & r_{33} \mid & t_{z}
\end{array}\right] \tilde{M}
$$

Since $s$ is the number that defines the scale of the objects with respect to their real size in the image. A is the matrix of intrinsic parameters that describes the position of the center of the image in pixels $(u 0, v 0)$. $[R \mid t]$ the matrix of extrinsic parameters of the camera that describe the rigid transformation (rotation and translation) between the coordinate system of the camera and the coordinate system of an object outside the camera. $\tilde{M}$ is a $3 \mathrm{D}$ point $(\mathrm{x}, \mathrm{y}, \mathrm{z})$ of the scene expressed in homogeneous coordinates. Subsequently, to achieve stereoscopic calibration, the translation vector that joins each camera reference system is calculated. The point position in three dimensions can be estimated from the coordinates in two dimensions and the Eq. 5.

$$
\left[\begin{array}{ccc}
u m_{31}-m_{11} & u m_{32}-m_{12} & u m_{33}-m_{13} \\
v m_{31}-m_{21} & v m_{31}-m_{22} & v m_{31}-m_{23} \\
u^{\prime} m_{31}^{\prime}-m_{31}^{\prime} & u^{\prime} m_{32}^{\prime}-m_{32}^{\prime} & u^{\prime} m_{31}^{\prime}-m_{31}^{\prime} \\
v^{\prime} m_{31}^{\prime}-m_{21}^{\prime} & v^{\prime} m_{31}^{\prime}-m_{32}^{\prime} & v^{\prime} m_{31}^{\prime}-m_{33}^{\prime}
\end{array}\right]\left[\begin{array}{c}
\hat{x} \\
\hat{y} \\
\hat{z}
\end{array}\right]=\left[\begin{array}{c}
m_{14}-u m_{34} \\
m_{24}-v m_{34} \\
m_{14}^{\prime}-u^{\prime} m_{34}^{\prime} \\
m_{24}^{\prime}-v^{\prime} m_{34}^{\prime}
\end{array}\right]
$$

Where $(u, v)$ and $\left(u^{\prime}, v^{\prime}\right)$ are the coordinates of the paired points obtained from the left and right cameras that correspond to the $3 \mathrm{D}$ point to be reconstructed.

\section{Results}

In Fig. 6 we present the reconstruction in the three dimensional space of all the points that correspond to the circle grid labels on the metallic sheet that were previously matched.

\subsection{Calculation of Superficial Strain Measurement on the Metal Sheet}

Punctual deformation was calculated by two methods. The first one consists only in the determination of how much each point was moved from a reference plane (plane in axis towards the cameras, where there is no deformation in the piece) to its current position. This provides a measure of how much each point of the material was stretched from one plane to the cameras. The drawback of this method is that it would not be able to measure the deformation correctly if it were produced in another direction or axis that was not the one that joins the piece to be measured and the reference camera. In Fig. 7, 


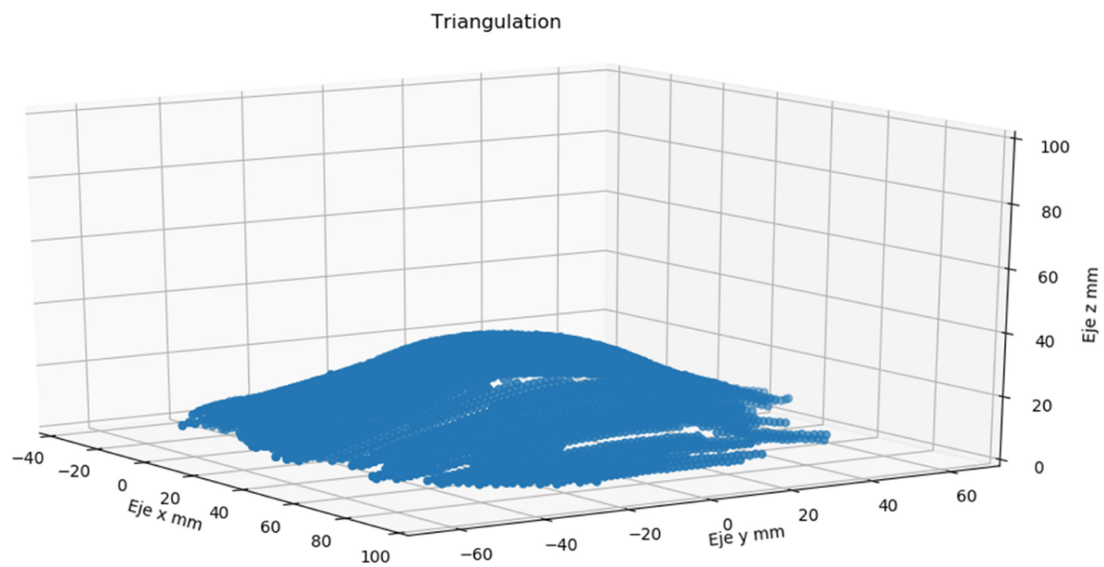

Fig. 6. 3D reconstruction of the metal sheet.

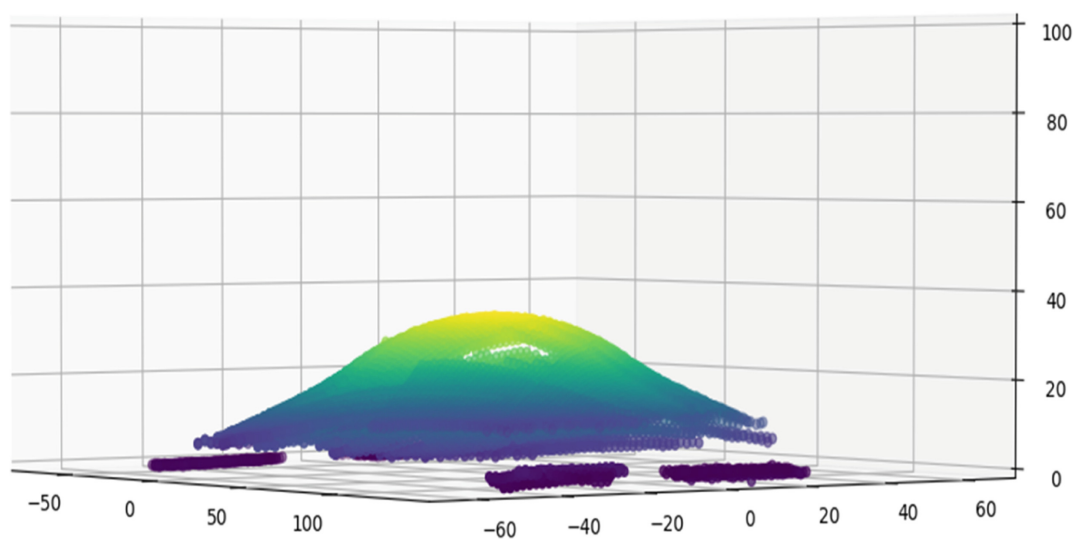

Fig. 7. Superficial strain measurement by the movement of each point on the $\mathrm{Z}$ axis.

it is observed in a color scale how the point deformation increases as the material moves away from the reference plane located on the $\mathrm{Z}$ axis with $\mathrm{z}=0 \mathrm{~cm}$.

The other developed method is to calculate the distance in the space between each point and its four neighbors and compare these values with measurements taken in the piece by the digital microscope, as in Fig. 8, where it is observed that there is an average distance of $1.55 \mathrm{~mm}$ among each circle of the grid (a), and about $1.7 \mathrm{~mm}$ after applying some deformation to the piece (b).

These values will serve as a comparative guide and demonstrate the expansion suffered by the surface when it is deformed. The distance among two points in 3D space is calculated using Eq. 6.

$$
d\left(\left(\begin{array}{l}
x_{1} \\
y_{1} \\
z_{1}
\end{array}\right),\left(\begin{array}{l}
x_{2} \\
y_{2} \\
z_{2}
\end{array}\right)\right)=\sqrt{\left(x_{1}-x_{2}\right)^{2}+\left(y_{1}-y_{2}\right)^{2}+\left(z_{1}-z_{2}\right)^{2}}
$$



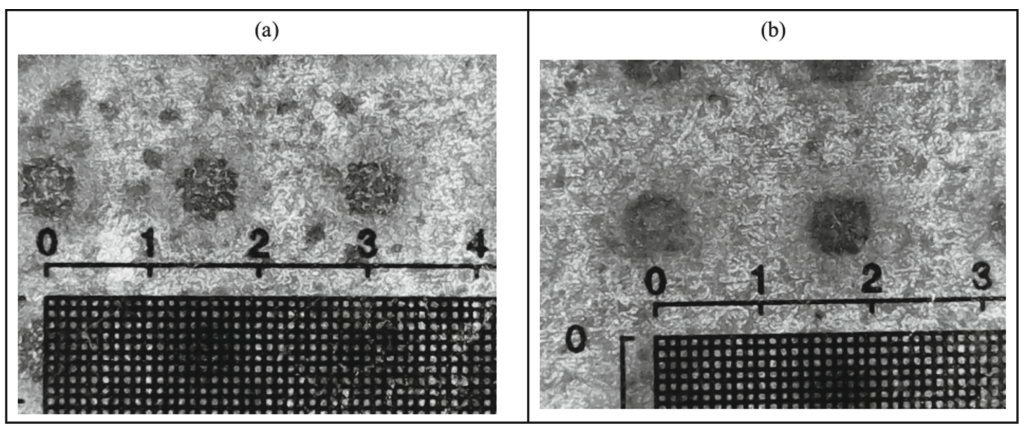

Fig. 8. (a) Measurement of the pattern on the metal sheet without deforming. (b) Measurement of the pattern in a deformed metal sheet.

In the Fig. 9 the deformation of each point is shown in color scale. The green circles have a distance between points less or equal to $1.55 \mathrm{~mm}$, yellows one varies from $1.56 \mathrm{~mm}$ to $1.63 \mathrm{~mm}$ and reds one are greater than $1.64 \mathrm{~mm}$. It shows a similarity with the previous method, the drawback would be the high computational cost required for the calculation of all points on the surface of the material.

Superficial strain measurement

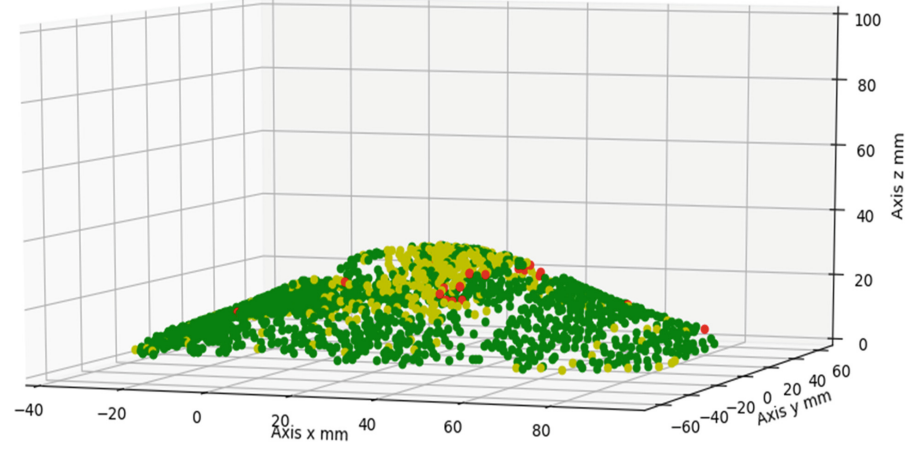

Fig. 9. Strain measurement by calculating the distance between each point and its neighbors.

Figure 10 shows the configurations of two groups of 5 points, one located on an area of the piece without deformation and another in the deformed area. The distance from the central point to each neighbor point in 3D space was calculated, the results are shown in Table 1 and 2 and compared with those obtained by the digital microscope. From these results, the average error in the experiment can be calculated, in this case $\varepsilon$ $=1.4451 \%$. 


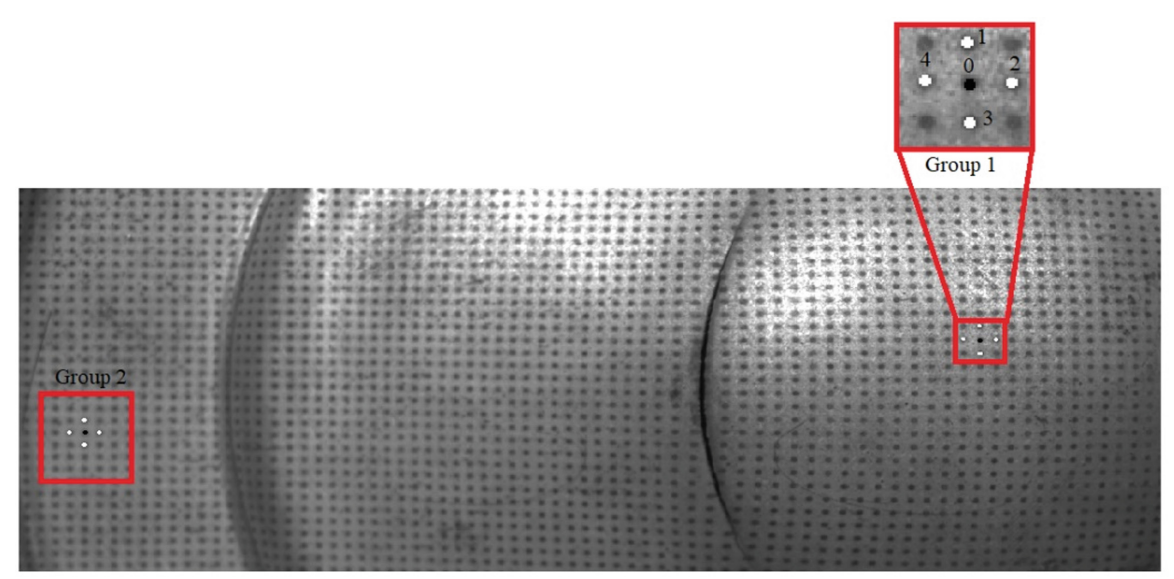

Fig. 10. Configuration of points to measure in planar and deformed regions.

Table 1. Comparison of the distances measured by the system and the microscope in Group 1.

\begin{tabular}{l|l|l|l|l}
\hline Group 1 & Point 1 & Point 2 & Point 3 & Point 4 \\
\hline $\begin{array}{l}\text { Distance to the Central Point measured by } \\
\text { the algorithm }\end{array}$ & $1.6468 \mathrm{~mm}$ & $1.6343 \mathrm{~mm}$ & $1.6436 \mathrm{~mm}$ & $1.6470 \mathrm{~mm}$ \\
\hline $\begin{array}{l}\text { Distance to the Central Point measured by } \\
\text { the microscope }\end{array}$ & $1.65 \mathrm{~mm}$ & $1.65 \mathrm{~mm}$ & $1.65 \mathrm{~mm}$ & $1.65 \mathrm{~mm}$ \\
\hline \begin{tabular}{l} 
Superficial Strain Measurement (\%) \\
\hline
\end{tabular} & 6.24 & 5.43 & 6.03 & 6.25 \\
\hline
\end{tabular}

Table 2. Comparison of the distances measured by the system and the microscope in Group 2.

\begin{tabular}{l|l|l|l|l}
\hline Group 2 & Point 1 & Point 2 & Point 3 & Point 4 \\
\hline $\begin{array}{l}\text { Distance to the Central Point measured by } \\
\text { the algorithm }\end{array}$ & $1.5276 \mathrm{~mm}$ & $1.5328 \mathrm{~mm}$ & $1.5758 \mathrm{~mm}$ & $1.5692 \mathrm{~mm}$ \\
\hline $\begin{array}{l}\text { Distance to the Central Point measured by } \\
\text { the microscope }\end{array}$ & $1.55 \mathrm{~mm}$ & $1.55 \mathrm{~mm}$ & $1.55 \mathrm{~mm}$ & $1.55 \mathrm{~mm}$ \\
\hline
\end{tabular}

\section{Discussion}

A great improvement in the performance of the developed algorithm is observed in Fig. 9 and Tables 1 and 2 compared with the method developed by Barranco (Ref. [11]). This is due to the fact that the area where the neighboring labels are sought is greater, giving possibility to correspond those labels that suffer a great deformation between one step and the next. Also, the search area is not one-dimensional, making it possible to search also for up and down displacements of neighboring labels. The new algorithm also needs less marks in both images matched manually to correspond the others, although it fails 
when it finds empty areas where labels of the marks stamped were not obtained due to bad image processing or due to presenting the piece oxidation spots. The Table 3 summarizes these comparative aspects.

Table 3. Comparison between methods for properly matched labels.

\begin{tabular}{l|l|l}
\hline & (Barranco et al., 2019) & (García et al., 2020) \\
\hline Initialization points quantity & 4 & 2 \\
\hline Total of founded correct labels & 4588 & 48556 \\
\hline
\end{tabular}

\section{Conclusion}

A method has been developed that allows correct triangulation and three-dimensional reconstruction of a metal sheet to calculate surface deformation. This method takes into account the problems existing in the matching of points by the Digital Image Correlation (quality of the speckle pattern, compromise between the measuring resolution and the large space for measuring to be done, time-consuming task related to the post-processing of the images, etc.). This is why a known pattern of circles was developed, allowing the 3D reconstruction of the sheet with a minimum of information, this provides more efficiency and a great benefit from the point of view of computational cost. Finally, DIC is used to corroborate the correspondence between points and verify the accuracy and efficiency of the developed method. The innovative part is the development of the semiautomatic matching algorithm, which allows to match all the points among both images using the labels that were obtained in the digital processing of the images. There is great similarity between the deformation values acquired by the algorithm and the digital microscope. The error in deformation measurements can vary according to many factors, including poor lighting conditions in the acquisition of images, incorrect digital processing of images, and even oxidation spots on the metal sheet that do not correspond to the pattern of drawn circles can contaminate and affect the results of point deformation.

\section{References}

1. Hilditch, T.B., de Souza, T., Hodgson, P.D.: Properties and automotive applications of advanced high-strength steels (AHSS). In: Shome, M., Tumuluru, M. (eds.) Welding and Joining of Advanced High Strength Steels (AHSS), pp. 9-28. Woodhead Publishing, Cambridge (2015)

2. Malesa, M., et al.: Non-destructive testing of industrial structures with the use of multi-camera digital image Correlation method. Eng. Fail. Anal. 69, 122-134 (2016)

3. Sutton, M.A., Orteu, J.J., Schreier, H.: Image correlation for shape, motion and deformation measurements: basic concepts, theory and applications. Springer, Boston (2009). https://doi. org/10.1007/978-0-387-78747-3 
4. Solav, D., Moerman, K.M., Jaeger, A.M., Genovese, K., Herr, H.M.: MultiDIC: An opensource toolbox for multi-view 3D digital image correlation. IEEE Access 6, 30520-30535 (2018)

5. Lecompte, D., et al.: Quality assessment of speckle patterns for digital image correlation. Opt. Lasers Eng. 44, 1132-1145 (2006)

6. Pan, B., Lu, Z., Xie, H.: Mean intensity gradient: an effective global parameter for quality assessment of the speckle patterns used in digital image correlation. Opt. Lasers Eng. 48, 469-477 (2010)

7. Genovese, K., Sorgente, D.: A morphing-based scheme for large deformation analysis with stereo-DIC. Opt. Lasers Eng. 104, 159-172 (2018)

8. Schreier, H.W., Sutton, M.A.: Systematic errors in digital image correlation due to undermatched subset shape functions. Exp. Mech. 42, 303-310 (2002). https://doi.org/10.1007/ BF02410987

9. Wang, Y., Sutton, M., Bruck, H., Schreier, H.: Quantitative error assessment in pattern matching: effects of intensity pattern noise, interpolation, strain and image contrast on motion measurements. Strain 45, 160-178 (2009)

10. Genovese, K., Casaletto, L., Rayas, J., Flores, V., Martinez, A.: Stereo-digital image correlation (DIC) measurements with a single camera using a biprism. Opt. Lasers Eng. 51, 278-285 (2013)

11. Barranco-Gutiérrez, A.-I., et al.: New four points initialization for digital image correlation in metal-sheet strain measurements. Appl. Sci. 9, 1691 (2019)

12. C Solutions: Short-course (2014)

13. Grediac, M., Frédéric, S., Benoît, B.: The grid method for in-plane displacement and strain measurement: a review and analysis. Strain 52(3), 205-243 (2016)

14. Badulescu, C., et al.: Applying the grid method and infrared thermography to investigate plastic deformation in aluminium multicrystal. Mech. Mater. 43(1), 36-53 (2011)

15. Zhou, M., Xie, H.: An identification method of mechanical properties of materials based on the full-field measurement method based on the fringe pattern. Strain 55(5), e12326 (2019)

16. Bomarito, G., Hochhalter, J., Ruggles, T., Cannon, A.: Increasing accuracy and precision of digital image correlation through pattern optimization. Opt. Lasers Eng. 91, 73-85 (2017)

17. Bruck, H., McNeill, S., Sutton, M.A., Peters, W.: Digital image correlation using NewtonRaphson method of partial differential correction. Exp. Mech. 29, 261-267 (1989). https:// doi.org/10.1007/BF02321405

18. Grediac, M., Blaysat, B., Sur, F.: A robust-to-noise deconvolution algorithm to enhance displacement and strain maps obtained with local DIC and LSA. Exp. Mech. 59, 219-243 (2019). https://doi.org/10.1007/s11340-018-00461-4

19. Koseki, K., Matsuo, T., Arikawa, S.: Measurement of super-pressure balloon deformation with simplified digital image correlation. Appl. Sci. 8, 2009 (2018)

20. Reu, P.L., et al.: DIC challenge: developing images and guidelines for evaluating accuracy and resolution of 2D analyses. Exp. Mech. 58, 1067-1099 (2018). https://doi.org/10.1007/ s11340-017-0349-0

21. Su, Y., Zhang, Q., Xu, X., Gao, Z.: Quality assessment of speckle patterns for DIC by consideration of both systematic errors and random errors. Opt. Lasers Eng. 86, 132-142 (2016)

22. Dai, X., et al.: The geometric phase analysis method based on the local high resolution discrete Fourier transform for deformation measurement. Measure. Sci. Technol. 25, 025402 (2014)

23. Bornert, M., et al.: Assessment of digital image correlation measurement errors: methodology and results. Exp. Mech. 49, 353-370 (2009). https://doi.org/10.1007/s11340-008-9204-7

24. Haddadi, H., Belhabib, S.: Use of rigid-body motion for the investigation and estimation of the measurement errors related to digital image correlation technique. Opt. Lasers Eng. 46, 185-196 (2008) 
25. Pan, B., Xie, H., Wang, Z., Qian, K., Wang, Z.: Study on subset size selection in digital image correlation for speckle patterns. Opt. Express 16, 7037-7048 (2008)

26. Yaofeng, S., Pang, J.H.: Study of optimal subset size in digital image correlation of speckle pattern images. Opt. Lasers Eng. 45, 967-974 (2007)

27. Di Lorenzo, E., Lava, P., Balcaen, R., Manzato, S., Peeters, B.: Full-field modal analysis using high-speed 3D digital image correlation. In: Journal of Physics: Conference Series, p. 012007. IOP Publishing (2018)

28. Tsai, R.: A versatile camera calibration technique for high-accuracy $3 \mathrm{D}$ machine vision metrology using off-the-shelf TV cameras and lenses. IEEE J. Robot. Autom. 3, 323-344 (1987)

29. Grediac, M., Blaysat, B., Sur, F.: Extracting displacement and strain fields from checkerboard images with the localized spectrum analysis. Exp. Mech. 59, 207-218 (2019). https://doi.org/ 10.1007/s11340-018-00439-2

30. Shao, X., Dai, X., Chen, Z., He, X.: Real-time 3D digital image correlation method and its application in human pulse monitoring. Appl. optics 55, 696-704 (2016)

31. Blaber, J., Adair, B., Antoniou, A.: Ncorr: open-source 2D digital image correlation matlab software. Exp. Mech. 55, 1105-1122 (2015). https://doi.org/10.1007/s11340-015-0009-1

32. Grédiac, M., Blaysat, B., Sur, F.: A critical comparison of some metrological parameters characterizing local digital image correlation and grid method. Exp. Mech. 57, 871-903 (2017). https://doi.org/10.1007/s11340-017-0279-x

33. Sur, F., Blaysat, B., Grediac, M.: Determining displacement and strain maps immune from aliasing effect with the grid method. Opt. Lasers Eng. 86, 317-328 (2016) 